\title{
Optimal design for active damping in sandwich structures using the Direct MultiSearch method
}

\author{
A.L. Araújo a , J.F.A. Madeira ${ }^{\text {b }}$, C.M. Mota Soares ${ }^{a, *}$, C.A. Mota Soares ${ }^{\text {a }}$ \\ a IDMEC, Instituto Superior Técnico, Universidade Técnica de Lisboa, Av. Rovisco Pais, 1049-001 Lisboa, Portugal \\ ${ }^{\mathrm{b}}$ ISEL - Instituto Superior de Engenharia de Lisboa, Rua Conselheiro Emídio Navarro 1, 1959-007 Lisboa, Portugal
}

\section{A R T I C L E I N F O}

\section{Article history:}

Available online 17 May 2013

\section{Keywords:}

Active damping

Piezoelectricity

Sandwich structures

Direct MultiSearch

Multiobjective optimization

\begin{abstract}
A B S T R A C T
This paper addresses the problem of optimal positioning of surface bonded piezoelectric patches in sandwich plates with viscoelastic core and laminated face layers. The objective is to maximize a set of modal loss factors for a given frequency range using multiobjective topology optimization. Active damping is introduced through co-located negative velocity feedback control. The multiobjective topology optimization problem is solved using the Direct MultiSearch Method. An application to a simply supported sandwich plate is presented with results for the maximization of the first six modal loss factors. The influence of the finite element mesh is analyzed and the results are, to some extent, compared with those obtained using alternative single objective optimization.
\end{abstract}

(c) 2013 Elsevier Ltd. All rights reserved.

\section{Introduction}

In this work we address a form of active constrained layer damping, where piezoelectric patch sensors and actuators are bonded to the exterior faces of a sandwich plate. The sandwich plate has composite laminated face layers and a core with viscoelastic material behavior. Piezoelectric patches are bonded to the surfaces of the sandwich plate and co-located control is assumed in order to provide active damping, using a negative velocity feedback control law.

In active damping it is important to be able to determine the optimal number of active control devices and their placement on the structure, as the number and placement of sensors can be critical to the robust functioning of active control systems. Also the size of actuators may be optimized in actuator placement problems, but the passive structure is always assumed to be of predetermined geometry and material. Pioneering works regarding the development of methodologies to determine the location of sensors and actuators using heuristic optimization techniques have been conducted by Franco Correia et al. [1], and Moita et al. [2], using simulated annealing algorithms. Previously, Suleman and Gonçalves [3] considered several objective functions simultaneously, such as maximizing the average static vertical

\footnotetext{
* Corresponding author. Tel.: +351 21841 7455; fax: +351 218417915 .

E-mail addresses: aurelio.araujo@ist.utl.pt (A.L. Araújo), jaguilar@dem.ist.utl.pt (J.F.A. Madeira), cristovao.mota.soares@ist.utl.pt (C.M. Mota Soares), carlosmotasoares@dem.ist.utl.pt (C.A. Mota Soares).
}

displacement of a beam and minimizing the mass of the actuators and minimizing the actuation voltage, where the design variables were the coordinates of actuator pairs and the size of rectangular actuator patches. Geometric constraints and upper and lower limits on design variables were considered. Adali et al. [4] also considered a beam problem where the maximum vertical deflection of a laminated beam was to be minimized using one pair of actuators, through a robust design approach. A practical application where this type of optimization is important is the design of helicopter rotor blades [5]. A state of the art review in optimization of smart structures and actuators has been conducted by Frecker [6] in 2003 and a review on optimization criteria for placement of piezoelectric sensors and actuators has been conducted by Gupta et al. [7] in 2010. More recently, Kiyono et al. [8] presented a topology optimization methodology for static design of piezolaminated shell structures by considering the optimization of piezoelectric material and polarization distributions together with the optimization of the fiber angle of the composite, considering three different objective functions for the design of actuators, sensors and energy harvesters.

In the present work we are concerned with the optimal placement of the co-located pairs of piezoelectric patch actuators in order to maximize modal loss factors in sandwich structures with viscoelastic core. For this purpose a topology optimization approach is considered and the multiobjective problem is solved using the Direct MultiSearch method. The obtained solutions are compared to the ones obtained through alternative methods [9] based on Genetic Algorithms. 


\section{Problem formulation}

\subsection{Finite element model}

A finite element model is used to obtain the dynamic response of the active sandwich plate of Fig. 1. The model is based on a mixed layerwise approach where the viscoelastic core $(v)$ is modeled according to a higher order shear deformation theory with cubic expansion of the in-plane displacement components in the thickness coordinate. The composite laminated face layers $\left(e_{1}, e_{2}\right)$ and the piezoelectric sensor $(s)$ and actuator $(a)$ layers are each modeled according to the first order shear deformation theory for laminated plates.

The basic assumptions in the development of the sandwich plate model are: all points on a normal to the plate have the same transverse displacement $w(x, y, t)$, where $t$ denotes time, and the origin of the $z$ axis is the medium plane of the core layer; no slip occurs at the interfaces between layers; the displacement is $C^{0}$ along the layer interfaces; transverse displacement is constant through the thickness of the sandwich; all materials are linear, homogeneous and orthotropic and the elastic layers $\left(e_{1}\right)$ and $\left(e_{2}\right)$ are made of laminated composite materials; for the viscoelastic core, material properties are complex and frequency dependent; upper and lower layers play the roles of sensor and actuator, respectively, and are connected via feedback control laws, considering co-located control.

The displacement field of the face layers may be written in the general form:

$$
\begin{aligned}
u^{i}(x, y, z, t) & =u_{0}^{i}(x, y, t)+\left(z-z_{i}\right) \theta_{x}^{i}(x, y, t) \\
v^{i}(x, y, z, t) & =v_{0}^{i}(x, y, t)+\left(z-z_{i}\right) \theta_{y}^{i}(x, y, t) \\
w^{i}(x, y, z, t) & =w_{0}(x, y, t)
\end{aligned}
$$

where $u_{0}^{i}$ and $v_{0}^{i}$ are the in-plane displacements of the mid-plane of the layer, $\theta_{x}^{i}$ and $\theta_{y}^{i}$ are rotations of normals to the mid-plane about the $y$ axis (anticlockwise) and $x$ axis (clockwise), respectively, $w_{0}$ is the transverse displacement of the layer (same for all layers in the sandwich), $z_{i}$ is the $z$ coordinate of the mid-plane of each layer, with reference to the core layer mid-plane $(z=0)$, and $i=s, e_{1}, e_{2}, a$ is the layer index.

For the viscoelastic core layer, the displacement field is written as a second order Taylor series expansion of the in-plane displacements in the thickness coordinate, with constant transverse displacement:

$$
\begin{aligned}
& u^{v}(x, y, z, t)=u_{0}^{v}(x, y, t)+z \theta_{x}^{v}(x, y, t)+z^{2} u_{0}^{* v}(x, y, t)+z^{3} \theta_{x}^{* v}(x, y, t) \\
& v^{v}(x, y, z, t)=v_{0}^{v}(x, y, t)+z \theta_{y}^{v}(x, y, t)+z^{2} v_{0}^{* v}(x, y, t)+z^{3} \theta_{y}^{* v}(x, y, t) \\
& w^{v}(x, y, z, t)=w_{0}(x, y, t)
\end{aligned}
$$

where $u_{0}^{v}$ and $v_{0}^{v}$ are the in-plane displacements of the mid-plane of the core, $\theta_{x}^{v}$ and $\theta_{y}^{v}$ are rotations of normals to the mid-plane of the core about the $y$ axis (anticlockwise) and $x$ axis (clockwise), respec-

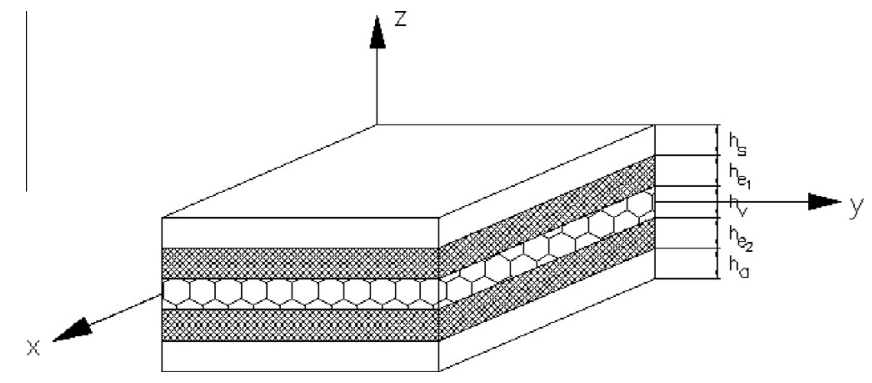

Fig. 1. Sandwich plate. tively, $w_{0}$ is the transverse displacement of the core (same for all layers in the sandwich). The functions $u_{0}^{* v}, v_{0}^{* v}, \theta_{x}^{* v}$ and $\theta_{y}^{* v}$ are higher order terms in the series expansion, defined also in the mid-plane of the core layer.

Displacement continuity at the layer interfaces is imposed, allowing us to retain the rotational degrees of freedom of the face layers, while eliminating the corresponding in-plane displacement ones. Hence, the generalized displacement field has 17 mechanical unknowns, where the vector of mechanical degrees of freedom is:

$$
d=\left\{\begin{array}{cccccccccccc}
\theta_{x}^{a} & \theta_{y}^{a} & \theta_{x}^{e_{2}} & \theta_{y}^{e_{2}} & u_{0}^{v} & v_{0}^{v} & w_{0} & \theta_{x}^{v} & \theta_{y}^{v} & u_{0}^{* v} & v_{0}^{* v} & \theta_{x}^{* v} \\
& \theta_{y}^{* v} & \theta_{x}^{e_{1}} & \theta_{y}^{e_{1}} & \theta_{x}^{s} & \theta_{y}^{s} & & & & & &
\end{array}\right\}^{T}
$$

We consider that fiber-reinforced laminae in elastic multi-layers $\left(e_{1}\right)$ and $\left(e_{2}\right)$, viscoelastic core $(v)$, and piezoelectric sensor $(s)$ and actuator $(a)$ layers are formulated as orthotropic. Furthermore, piezoelectric material is assumed to be $m m 2$ orthorhombic, polarized in the thickness direction. Constitutive equations for each lamina in the sandwich may then be expressed in the principal material directions, and for the zero transverse normal stress situation as $[10,11]$ :

$$
\begin{aligned}
& \left\{\begin{array}{l}
\sigma_{11} \\
\sigma_{22} \\
\sigma_{23} \\
\sigma_{13} \\
\sigma_{12}
\end{array}\right\}=\left[\begin{array}{ccccc}
Q_{11} & Q_{12} & 0 & 0 & 0 \\
Q_{12} & Q_{22} & 0 & 0 & 0 \\
0 & 0 & Q_{44} & 0 & 0 \\
0 & 0 & 0 & Q_{55} & 0 \\
0 & 0 & 0 & 0 & Q_{66}
\end{array}\right]^{E}\left\{\begin{array}{l}
\varepsilon_{11} \\
\varepsilon_{22} \\
\gamma_{23} \\
\gamma_{13} \\
\gamma_{12}
\end{array}\right\} \\
& -\left[\begin{array}{ccc}
0 & 0 & e_{31}^{*} \\
0 & 0 & e_{32}^{*} \\
0 & e_{24} & 0 \\
e_{15} & 0 & 0 \\
0 & 0 & 0
\end{array}\right]\left\{\begin{array}{l}
\mathcal{E}_{1} \\
\mathcal{E}_{2} \\
\mathcal{E}_{3}
\end{array}\right\} \\
& \left\{\begin{array}{l}
D_{1} \\
D_{2} \\
D_{3}
\end{array}\right\}=\left[\begin{array}{ccccc}
0 & 0 & 0 & e_{15} & 0 \\
0 & 0 & e_{24} & 0 & 0 \\
e_{31}^{*} & e_{32}^{*} & 0 & 0 & 0
\end{array}\right]\left\{\begin{array}{l}
\varepsilon_{11} \\
\varepsilon_{22} \\
\gamma_{23} \\
\gamma_{13} \\
\gamma_{12}
\end{array}\right\}+\left[\begin{array}{ccc}
\epsilon_{11} & 0 & 0 \\
0 & \epsilon_{22} & 0 \\
0 & 0 & \epsilon_{33}^{*}
\end{array}\right]^{\varepsilon}\left\{\begin{array}{l}
\mathcal{E}_{1} \\
\mathcal{E}_{2} \\
\mathcal{E}_{3}
\end{array}\right\}
\end{aligned}
$$

where $\sigma_{i j}$ are stress components, $\varepsilon_{i j}$ and $\gamma_{i j}$ are strain components, $\mathcal{E}_{i}$ and $D_{i}$ are the electric field and electric displacement components, respectively, $Q_{i j}^{E}$ are reduced stiffness coefficients at constant electric field, $e_{i j}$ and $e_{i j}^{*}$ are piezoelectric and reduced piezoelectric constants, respectively, and $\epsilon_{i j}^{\varepsilon}$ and $\epsilon_{33}^{* \varepsilon}$ are dielectric and reduced dielectric constants, measured at constant strain. Expressions for the reduced quantities mentioned above can be found in Araújo et al. [10] and Benjeddou and Dëu [11]. The complete set of equations in (4) is only used for the piezoelectric sensor and actuator layers, while for the remaining elastic and viscoelastic layers, only the first equation is considered, without the piezoelectric part. For the viscoelastic core layer, the reduced stiffness coefficients $Q_{i j}^{E}$ are complex quantities, since the complex modulus approach was used in this work, using the elastic-viscoelastic principle. In this case, the usual engineering moduli may be represented by complex quantities, considering isothermal conditions:

$$
\begin{aligned}
& E_{1}^{*}(j \omega)=E_{1}(\omega)\left(1+j \eta_{E_{1}}(\omega)\right) \\
& E_{2}^{*}(j \omega)=E_{2}(\omega)\left(1+j \eta_{E_{2}}(\omega)\right) \\
& G_{12}^{*}(j \omega)=G_{12}(\omega)\left(1+j \eta_{G_{12}}(\omega)\right) \\
& G_{23}^{*}(j \omega)=G_{23}(\omega)\left(1+j \eta_{G_{23}}(\omega)\right) \\
& G_{13}^{*}(j \omega)=G_{13}(\omega)\left(1+j \eta_{G_{13}}(\omega)\right) \\
& v_{12}^{*}(j \omega)=v_{12}(\omega)\left(1+j \eta_{v_{12}}(\omega)\right)
\end{aligned}
$$


where $E_{1}, E_{2}, G_{12}, G_{23}, G_{13}$ and $v_{12}$ denote storage moduli, $\eta_{E_{1}}, \eta_{E_{2}}, \eta_{G_{12}}, \eta_{G_{23}}, \eta_{G_{13}}$ and $\eta_{v_{12}}$ are the corresponding material loss factors, $\omega$ represents the angular frequency of vibration and $j=\sqrt{-1}$ is the imaginary unit. Additionally, in Eq. (5), E, $G$ and $v$ represent Young's moduli, shear moduli and Poisson's ratio, respectively.

The equations of motion for the plate are obtained by applying the generalized Hamilton's principle, using an eight node serendipity plate element with 17 mechanical degrees of freedom per node as presented in Eq. (3), and one electric potential degree of freedom per piezoelectric layer. It is assumed that the electric potential varies linearly in the thickness direction and compatibility of displacements at the layer interfaces is enforced:

$$
\left[\begin{array}{cc}
\mathbf{M}_{u u} & \mathbf{0} \\
\mathbf{0} & \mathbf{0}
\end{array}\right]\left[\begin{array}{l}
\ddot{\mathbf{u}} \\
\ddot{\boldsymbol{\phi}}
\end{array}\right]+\left[\begin{array}{ll}
\mathbf{K}_{u u}(\omega) & \mathbf{K}_{u \phi} \\
\mathbf{K}_{u \phi}^{T} & \mathbf{K}_{\phi \phi}
\end{array}\right]\left[\begin{array}{l}
\mathbf{u} \\
\boldsymbol{\phi}
\end{array}\right]=\left[\begin{array}{c}
\mathbf{F}_{u} \\
\mathbf{0}
\end{array}\right]
$$

where $\mathbf{u}, \ddot{\mathbf{u}}, \phi$ and $\ddot{\phi}$ are complex mechanical degrees of freedom and corresponding accelerations, electric potential and corresponding second time derivatives, respectively. $\mathbf{M}_{u u}$ and $\mathbf{K}_{u u}(\omega)$ are the real mass matrix and complex stiffness matrix, respectively, corresponding to purely mechanical behavior, while $\mathbf{K}_{\phi \phi}$ is the dielectric stiffness matrix, $\mathbf{K}_{u \phi}$ is the stiffness matrix that corresponds to the coupling between the mechanical and the piezoelectric effects, and $\mathbf{F}_{u}$ is the externally applied complex mechanical load vector.

The feedback control law is based on direct proportional or velocity feedback, and can be written in the following form:

$\phi_{a}=G_{d} \phi_{s}+G_{v} \dot{\phi}_{s}$

where $G_{d}$ and $G_{v}$ are the constant displacement and the constant velocity feedback gains, respectively. The vectors of actuator (a) and sensor (s) potentials are $\phi_{a}$ and $\phi_{s}$, while $\dot{\phi}_{s}$ is the vector of sensor potential time derivatives.

Assuming harmonic vibrations, the final equilibrium equations are given by:

$\left[\mathbf{K}^{*}(\omega)-\omega^{2} \mathbf{M}_{u u}\right] \mathbf{u}=\mathbf{F}_{u}$

where the condensed stiffness matrix is written as:

$\mathbf{K}^{*}(\omega)=\mathbf{K}_{u u}(\omega)-\left[\left(G_{d}+j \omega G_{v}\right) \mathbf{K}_{u \phi}^{a}+\mathbf{K}_{u \phi}^{s}\right] \mathbf{K}_{\phi \phi}^{s^{-1}} \mathbf{K}_{u \phi}^{s^{\top}}$

and $\mathbf{K}_{u u}(\omega)$ is a complex matrix and $\mathbf{F}_{u}$ is the Fourier transform of the time domain force history.

It is worthwhile noting that when electroded surfaces exist in a given patch or layer, equipotential conditions should be imposed before condensing the electric degrees of freedom. The forced vibration problem is solved in the frequency domain, which requires the solution of the linear system of equations in Eq. (8) for each frequency point.

For the free vibration problem, Eq. (8) reduces to the following nonlinear eigenvalue problem, due to the frequency dependent nature of the stiffness matrix:

$\left[\mathbf{K}^{*}(\omega)-\lambda_{n}^{*} \mathbf{M}_{u u}\right] \mathbf{u}_{n}=\mathbf{0}$

where $\mathbf{u}_{n}$ is a complex eigenvector and $\lambda_{n}^{*}$ is the associated complex eigenvalue, which can be written as:

$\lambda_{n}^{*}=\lambda_{n}\left(1+j \eta_{n}\right)$

and $\lambda_{n}=\omega_{n}^{2}$ is the real part of the complex eigenvalue and $\eta_{n}$ is the corresponding modal loss factor. The nonlinear eigenvalue problem is solved iteratively and the iterative process is considered to have converged when:

$\frac{\left\|\omega_{i}-\omega_{i-1}\right\|}{\omega_{i-1}} \leqslant \epsilon$

where $\omega_{i}$ and $\omega_{i-1}$ are current and previous iteration values for the real part of the particular eigenfrequency of interest, respectively, and $\epsilon$ is the convergence tolerance. Further details regarding the model can be found in Araújo et al. [12].

\subsection{Optimal design formulation}

A constrained nonlinear multiobjective optimization problem can be mathematically formulated as

$$
\begin{aligned}
& \max F(x) \equiv\left(f_{1}(x), f_{2}(x), \ldots, f_{m}(x)\right)^{\top} \\
& \text { s.t. } x \in \Omega
\end{aligned}
$$

involving $m$ objective functions $f_{j}: \Omega \subseteq \mathbb{R}^{n} \rightarrow \mathbb{R} \cup\{+\infty\}, j=1, \ldots, m$ to maximize. Recall that to maximize $f_{j}$ is equivalent to minimize $-f_{j .} \emptyset \neq \Omega \subseteq \mathbb{R}^{n}$ represents the feasible region.

In the presence of $m(\geqslant 2)$ objective functions, the maximizer of one function is not necessarily the maximizer of another. In this case, we do not have a single point that yields the "optimum point for all objectives". Instead, we have a set of points, called Pareto optimal or nondominated set. Given two points $x_{1}, x_{2}$ in $\Omega, x_{1}$ is said to dominate, in Pareto sense, another point $x_{2}$ if and only if solution $x_{1}$ is strictly better than $x_{2}$ in at least one of the objectives and point $x_{1}$ is not worse than $x_{2}$ in any of the objectives. A set of points in $\Omega$ is nondominated when no point in the set is dominated by another one in the set.

Direct MultiSearch (DMS) [13] is a solver for multiobjective optimization problems, without the use of derivatives and does not aggregate any components of the objective function. It essentially generalizes all direct-search methods of directional type from single to multiobjective optimization. DMS maintains a list of feasible nondominated points. At each iteration, the new feasible evaluated points are added to this list and the dominated ones are removed. Successful iterations correspond then to an iterate list changes, meaning that a new feasible nondominated point was found. Otherwise, the iteration is declared as unsuccessful.

The problem of finding the optimal distribution of a given number of piezoelectric sensor/actuator pairs for maximum damping is formulated as a multiobjective topology optimization problem:

$$
\begin{array}{rl}
\max _{\rho_{e}} & F\left(\rho_{e}\right) \equiv\left(f_{1}\left(\rho_{e}\right), f_{2}\left(\rho_{e}\right), \ldots, f_{m}\left(\rho_{e}\right)\right)^{\top} \\
\text { s.t. } & V \leqslant V_{0} \\
& \rho_{\min } \leqslant \rho_{e} \leqslant 1
\end{array}
$$

where $f_{i}\left(\rho_{e}\right)=\eta_{i}, \eta_{i}$ are modal loss factors obtained by iteratively solving the nonlinear eigenvalue problem in Eq. (10), $\rho_{e}$ are the design variables, corresponding to the pseudo-densities of the piezoelectric material in each element $(e)$ of the finite element mesh, $V$ is the total volume of piezoelectric material and $V_{0}$ is the maximum allowable volume of piezoelectric material. The formulation used in this work is based on the PEMAP (piezoelectric material with penalization) model [14], where the material pseudo-density $\rho_{e}$ of each piezoelectric patch element is used to achieve a continuous material model [15]:

$\mathbf{K}_{u u}^{(e)}=\rho_{e}^{p_{K}} \mathbf{K}_{\mathbf{0}_{u u}^{(e)}}^{(e)}$

$\mathbf{K}_{u \phi}^{(e)}=\rho_{e}^{p_{K}} \mathbf{K}_{\mathbf{0}_{u \phi}^{(e)}}^{(e)}$

$\mathbf{M}_{u u}^{(e)}=\rho_{e}^{p_{M}} \mathbf{M}_{\mathbf{0}_{u}}^{(e)}$

where $\mathbf{K}_{\mathbf{0}_{u u}}^{(e)}, \mathbf{K}_{\mathbf{0}_{u},}^{(e)}$ and $\mathbf{M}_{\mathbf{0}_{u u}}^{(e)}$ are the element matrices for the piezoelectric material, without penalization. Two penalization factors, $p_{K}$ and $p_{M}$, are used to penalize intermediate densities, easing the task of obtaining a near discrete $0-1$ solution. In this work $p_{K}=3$ and $p_{M}=1$ are used for the stiffness and the mass of the piezoelectric patches. It should be noted that all the element matrices in Eq. (15) refer only to the piezoelectric patches: the base sandwich plate is never affected by the pseudo-density variables. 


\section{Application}

The plate considered for the application is a simply supported $300 \times 200 \mathrm{~mm}$ composite laminated sandwich plate with a $1 \mathrm{~mm}$ thick core and with face layers made of three $0.5 \mathrm{~mm}$ thick layers, with lamination scheme $\left[90^{\circ}{ }_{e} / 45^{\circ}{ }_{e} / 45^{\circ}{ }_{e} / 0^{\circ}{ }_{v} / 45^{\circ}{ }_{e} / 45^{\circ}{ }_{e} / 90^{\circ}{ }_{e}\right.$ ], where subscripts $v$ and $e$ stand for viscoelastic core and elastic face layers, respectively. Simply supported conditions are applied in such a way that all the translational degrees of freedom at the border of the plate are constrained.

The piezoelectric patches to be used have surface electrodes and in-plane dimensions of $50 \times 50 \mathrm{~mm}$ and are $0.1 \mathrm{~mm}$ thick, and only four pairs of patches are available to be bonded to the composite plate, corresponding to a maximum allowable volume of $V_{0}=2000 \mathrm{~mm}^{3}$. A negative velocity feedback control law with gain $G_{v}=-0.01$ was used for each pair of co-located sensor and actuator.

Material properties are presented in Table 1. For the present application the core is isotropic and the piezoelectric patches are transversely isotropic. Although the problem has been formulated considering a viscoelastic core, it should be noted that the material loss factor for the viscoelastic core is disregarded in the present analysis, since we are concentrated on maximizing active damping and passive damping has an additive effect.

The objective will be to determine the best location for these four pairs of co-located sensors and actuators, in order to maximize the first six modal loss factors of the bending modes.

\subsection{Coarse mesh}

The first step was to discretize the plate in a coarse $6 \times 4$ finite element mesh. Nondominated solutions were obtained and from this set, the solutions that maximize individually the first six modal loss factors are presented in Fig. 2. We can observe that the configuration that maximizes objectives 1,3 and 4 is the same. The best solution in terms of the maximization of the weighted sum of the first six modes $\sum_{i=1}^{6} \mathbf{w}_{i} \eta_{i}$, with linearly decreasing weights $\left(w_{1}=0.323, w_{2}=0.261, w_{3}=0.198, w_{4}=0.135, w_{5}=0.073\right.$ and $\left.w_{6}=0.01\right)$ is also presented in Fig. 3. In all the figures shaded elements correspond to the patch positions and, for each configuration, the value of the corresponding modal loss factor is shown.

It is worthwhile noting that the design variables in the Direct MultiSearch solver are continuous $\left(0.1 \leqslant \rho_{e} \leqslant 1\right)$, where the lower limit is nonzero for numerical stability reasons in the finite element analysis. However, before an evaluation of the objectives is conducted, all the design variables are rounded to their limits (i.e., if $\rho_{e} \geqslant 0.5$ then $\rho_{e}=1$ and if $\rho_{e}<0.5$ then $\rho_{e}=0.1$ ) and the volume constraint is then verified. This allows us to transform the problem with continuous design variables into one with discrete design variables.

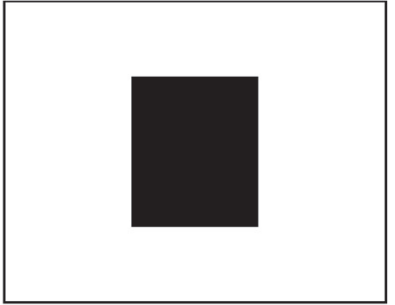

(a) $\eta_{1}=14.27 \%$

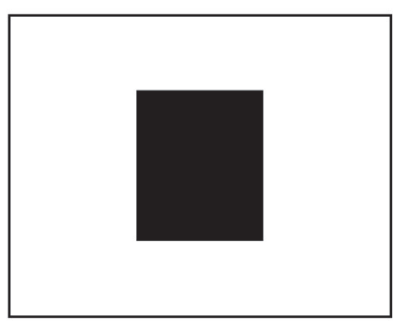

(c) $\eta_{3}=36.34 \%$

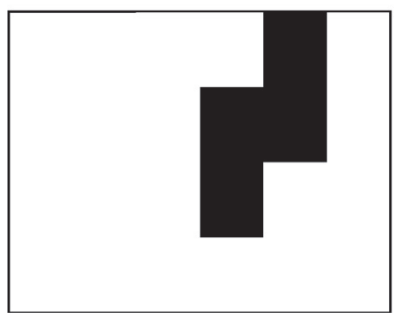

(e) $\eta_{5}=32.45 \%$

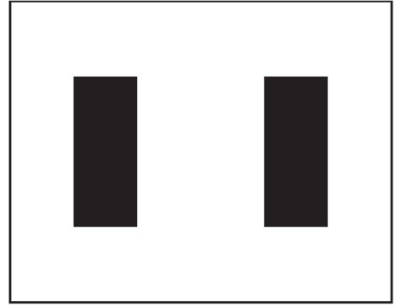

(b) $\eta_{2}=29.50 \%$

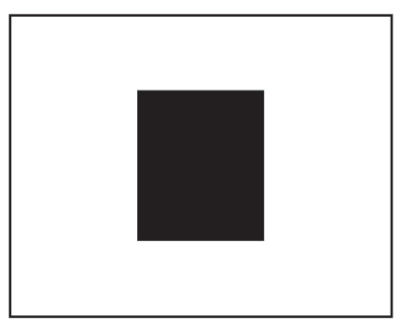

(d) $\eta_{4}=13.62 \%$

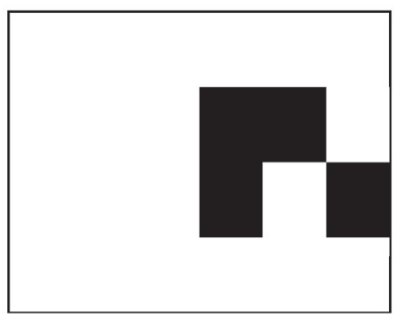

(f) $\eta_{6}=50.02 \%$
Fig. 2. Best solutions for individual objectives with the coarse $6 \times 4$ mesh.

For comparison purposes, a Genetic Algorithm [16] with binary encoding [17] is also used to solve the problem of maximizing the weighted sum of the first 6 modal loss factors. The algorithm initializes a random sample of individuals with different parameters to be optimized using evolution via survival of the fittest. The selection scheme used is tournament selection with a shuffling technique for choosing random pairs for mating. The micro-GA option has been used, with uniform crossover and elitism (the best individual is always replicated into the next generation). A population size of 10 individuals was used for the Genetic Algorithm, with a maximum of 200 generations, crossover probability of 0.5 and mutation probability of 0.02 . The optimal configuration obtained was the same as in Fig. 3, which allows us to confirm our solution using the nondominated solutions obtained through DMS.

Fig. 4 presents the magnitude of the frequency response functions for the configuration of Fig. 3, obtained at a point located at the center of the top rightmost quarter of the plate, with an impul-

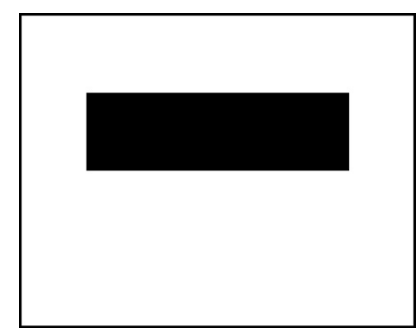

Fig. 3. Best solution for weighted sum of objectives $\left(\sum_{i=1}^{6} \mathbf{w}_{i} \eta_{i}=17.77 \%\right)$ with the coarse $6 \times 4$ mesh.
Table $\mathbf{1}$
Material properties.

\begin{tabular}{lllc}
\hline & Elastic layers & Core & Patches \\
\hline$E_{1}(\mathrm{GPa})$ & 98.0 & $59.6 \times 10^{-3}$ & 47.0 \\
$E_{2}(\mathrm{GPa})$ & 7.9 & $59.6 \times 10^{-3}$ & 47.0 \\
$G_{12}(\mathrm{GPa})$ & 5.6 & $20 \times 10^{-3}$ & 16.3 \\
$G_{13}(\mathrm{GPa})$ & 5.6 & $20 \times 10^{-3}$ & 17.4 \\
$G_{23}(\mathrm{GPa})$ & 5.6 & $20 \times 10^{-3}$ & 17.4 \\
$v_{12}$ & 0.28 & 0.49 & 0.33 \\
$e_{31}^{*}(\mathrm{~N} / \mathrm{Vm})$ & - & - & -14.7 \\
$e_{32}^{*}(\mathrm{~N} / \mathrm{Vm})$ & - & - & -14.7 \\
$\epsilon_{33}^{* \varepsilon}\left(\times 10^{-9} \mathrm{~F} / \mathrm{m}\right)$ & - & - & 21.2 \\
$\rho\left(\mathrm{kg} / \mathrm{m}^{3}\right)$ & 1520 & 1140 & 8036 \\
\hline
\end{tabular}




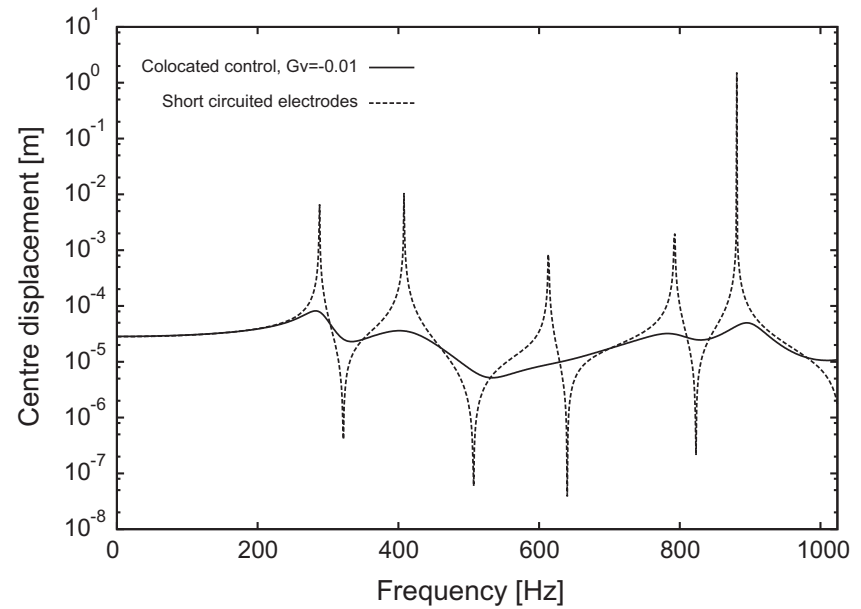

Fig. 4. Frequency response function (magnitude) for the solution of Fig. 3, with and without control.

sive excitation force of $10 \mathrm{~N}$, applied at the same point. The amplitude reduction for the considered frequency range is evident, demonstrating the effectiveness of active control.

\subsection{Refined mesh}

As it is understood that a coarse mesh will bias the solution, the next step was to use a more refined $12 \times 8$ finite element mesh. In order to be able to use this mesh and at the same time maintain the size of the sensors and actuators, equipotential conditions have to be imposed to the finite elements belonging to the same patch. Hence, it is no longer possible to have the same simple procedure as before, since all the piezoelectric elements have to be grouped in as many sets as the available patches (in this case there will be four groups of four elements). The number of design variables is equal to the number of elements in the finite element mesh $(12 \times 8=96)$ and there will be four patches, each with $2 \times 2$ elements. The following algorithm was implemented to guarantee

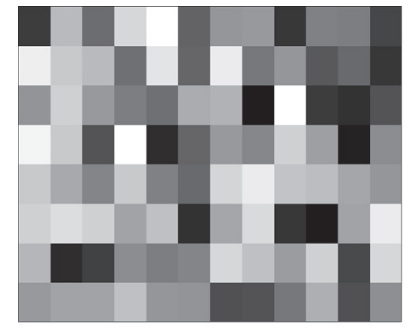

(a) Initial

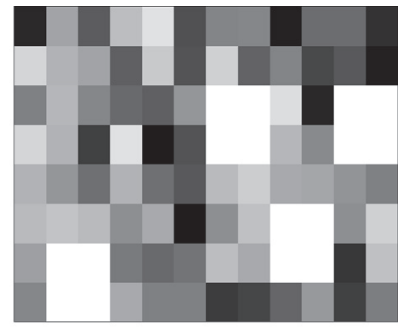

(b) Intermediate

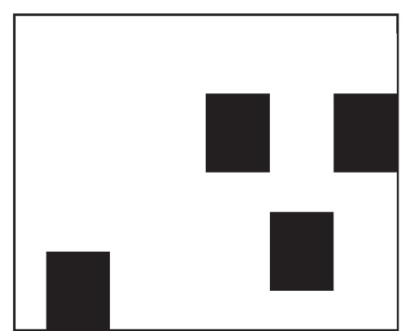

(c) Final

Fig. 5. Example of application of the algorithm for selecting patch positions with the refined mesh.

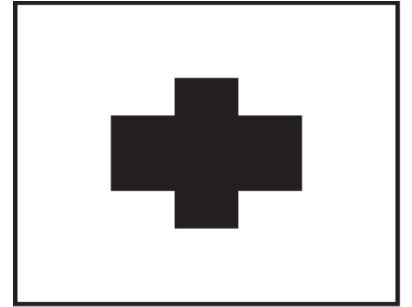

(a) $\eta_{1}=15.06 \%$

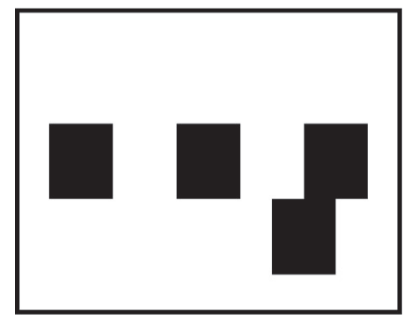

(c) $\eta_{3}=46.15 \%$

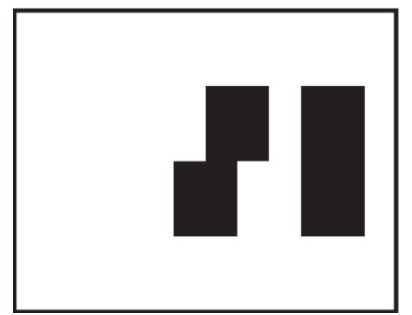

(e) $\eta_{5}=60.69 \%$

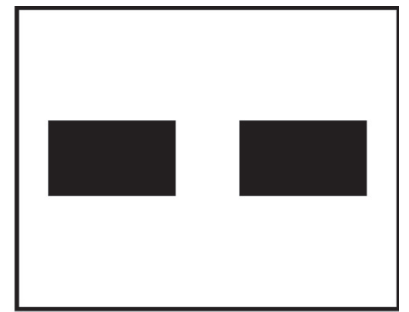

(b) $\eta_{2}=33.54 \%$

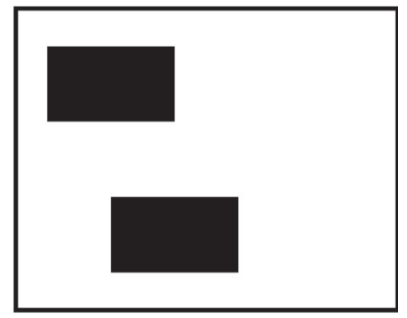

(d) $\eta_{4}=43.17 \%$

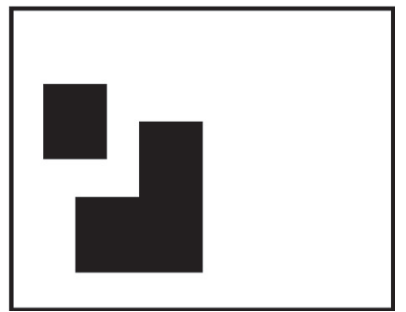

(f) $\eta_{6}=62.56 \%$
Fig. 6. Best solutions for individual objectives with the $12 \times 8$ mesh.

that only solutions with the correct grouping of elements are evaluated:

1. A search for the element with the maximum density is conducted.

2. The patch position is then chosen among the four possible locations in the neighborhood of the selected element. The maximum product of densities of the elements that make up the candidate patches is the selection criteria.

3. The densities of the four elements of the selected patch are set to zero and the process is repeated for the selection of the remaining patches.

4. In the end, all elements with zero density are set to unit density and the remaining ones to the minimum density.

5. Equipotential conditions are imposed to the four elements of each patch and the solution is evaluated.

An example of application of this algorithm is presented in Fig. 5, where from an initial continuous solution, an intermediate solution is obtained by applying four times the first three steps of the algorithm, and the final solution is obtained by applying step four of the algorithm.

Using this algorithm along with our optimization problem, nondominated solutions were obtained and from this set, the solutions that maximize individually the first six modal loss factors are presented in Fig. 6. Furthermore, the best solution from the nondominated set that maximizes the weighted sum of the first six modal loss factors is also presented in Fig. 7.

Again, Fig. 8 presents the magnitude of the frequency response functions for the configuration of Fig. 7, obtained at a point located at the center of the top rightmost quarter of the plate, with an 


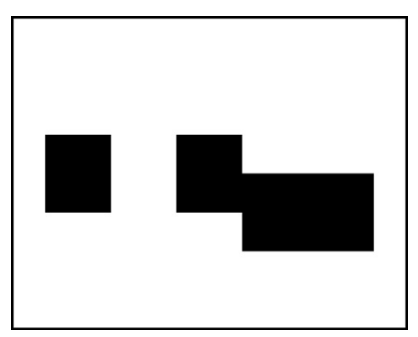

Fig. 7. Best solution for weighted sum of objectives $\left(\sum_{i=1}^{6} \mathbf{w}_{i} \eta_{i}=17.42 \%\right)$ with the $12 \times 8$ mesh.

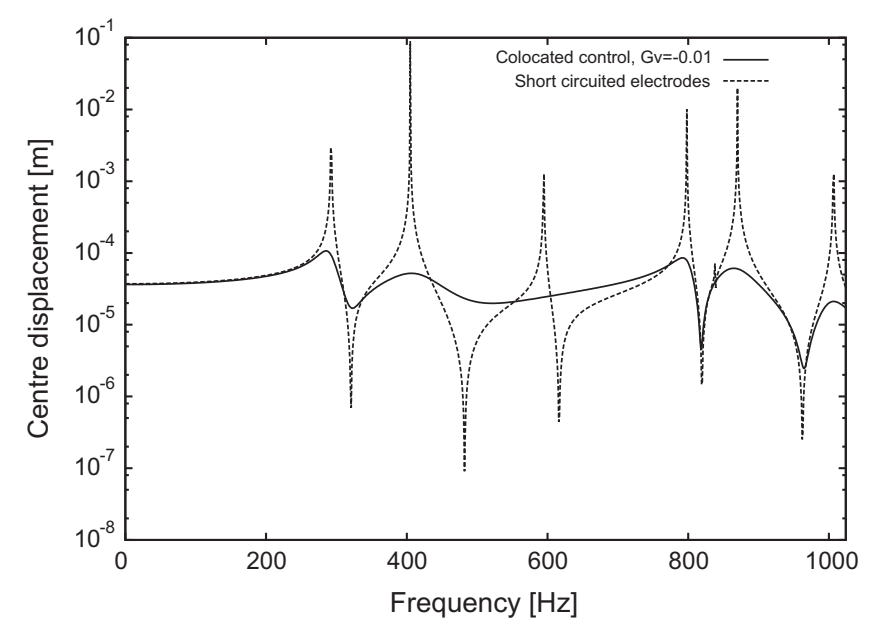

Fig. 8. Frequency response function (magnitude) for the solution of Fig. 7, with and without control.

impulsive excitation force of $10 \mathrm{~N}$, applied at the same point. The amplitude reduction for the considered frequency range is evident, demonstrating the effectiveness of active control.

\section{Discussion of results}

A comparison of the results of the refined mesh with the ones obtained with the coarse mesh shows the influence of the mesh size. The solutions obtained with the refined mesh correspond to positions of the patches that are not possible to obtain using the coarse mesh. One can also notice that the best solution obtained with the refined mesh for the weighted sum of objectives has a lower value of the objective function when compared with the corresponding solution using the coarse mesh. This is definitely due to the mesh size influence on the results, as a check was done using the optimal positioning of Fig. 3 along with the refined mesh, resulting in $\sum_{i=1}^{6} \mathbf{w}_{i} \eta_{i}=16.84 \%$, which is in fact a worse solution than the one obtained in Fig. 7, with respect to this objective.

When comparing the frequency response functions using both coarse and refined meshes, as displayed in Figs. 4 and 8, respectively, one observes that a substantial attenuation is obtained with the application of negative feedback control laws in both cases. The dotted curves in these figures represent the frequency response of the plates with the patches in each of the obtained optimal configurations of Figs. 3 and 7, but with short circuited electrodes, hence eliminating the piezoelectric effect. Although substantially higher modal loss factors are obtained for some modes with the refined mesh, from the frequency response functions one can observe that the reduction in the amplitudes of vibration are equivalent for both meshes, and that from this point of view the coarse mesh gives also acceptable results, from an engineering point of view.

\section{Conclusions}

A multiobjective approach to the optimal positioning of piezoelectric patches in sandwich plates for active damping maximization has been presented. Collocated negative velocity feedback control has been used to provide active damping. Nondominated solutions have been obtained with the Direct MultiSearch solver, using the first six modal loss factors as objectives to maximize. From these nondominated solutions, those that maximize individual objectives and also a weighted sum of objectives are presented and discussed for two types of finite element meshes, showing the expected influence of the mesh size in the optimal designs. Frequency response functions show the effectiveness of active damping in the response of the optimal structures with substantial reduction in the vibration levels.

\section{Acknowledgments}

This work has been supported by National Funds through Fundação para a Ciência e Tecnologia (FCT), Projects PEst-OE/ EME/LA0022/2011 and FCT-PTDC/EME/120830/2010.

\section{References}

[1] Franco Correia VM, Mota Soares CM, Mota Soares CA. Buckling optimization of composite laminated adaptive structures. Compos Struct 2003;62:315-21.

[2] Moita JM, Franco Correia VM, Martins PG, Mota Soares CM, Mota Soares CA. Optimal design in vibration control of adaptive structures using a simulated annealing algorithm. Compos Struct 2006;75:79-87.

[3] Suleman A, Gonçalves MA. Multi-objective optimization of an adaptive composite beam using the physical programming approach. J Intell Mater Syst Struct 1999;10:56-70.

[4] Adali SJ, Bruch JC, Sadek IS, Sloss JM. Robust shape control of beams with load uncertainties by optimally placed piezo actuators. Struct Multidisciplinary Optim 2000;19:274-81.

[5] Glukhikh S, Barkanov E, Kovalev A, Masarati P, Morandini M, Riemenschneider $\mathrm{J}$, et al. Design of helicopter rotor blades with actuators made of a piezomacrofiber composite. Mech Compos Mater 2008;44:57-64.

[6] Frecker MI. Recent advances in optimization of smart structures and actuators. J Intell Mater Syst Struct 2003;14:207-16.

[7] Gupta V, Sharma M, Thakur N. Optimization criteria for optimal placement of piezoelectric sensors and actuators on a smart structure: a technical review. J Intell Mater Syst Struct 2010;21:1227-43.

[8] Kiyono CY, Silva ECN, Reddy JN. Design of laminated piezocomposite shell transducers with arbitrary fiber orientation using topology optimization approach. Int J Numer Methods Eng 2012;90:1452-84.

[9] Araújo AL, Martins P, Mota Soares CM, Mota Soares CA, Herskovits J. Damping optimisation of hybrid active-passive sandwich composite structures. Adv Eng Software 2012;46:69-74.

[10] Araújo AL, Lopes HMR, Vaz MAP, Mota Soares CM, Herskovits J, Pedersen P. Parameter estimation in active plate structures. Comput Struct 2006;84:1471-9.

[11] Benjeddou A, Dëu JF. A two-dimensional closed-form solution for the freevibrations analysis of piezoelectric sandwich plates. Int J Solids Struct 2002;39:1463-86.

[12] Araújo AL, Mota Soares CM, Mota Soares CA, C A. Finite element model for hybrid active-passive damping analysis of anisotropic laminated sandwich structures. J Sandwich Struct Mater 2010;12:397-419.

[13] Custódio AL, Madeira JFA, Vaz AIF, Vicente LN. Direct MultiSearch for multiobjective optimization. SIAM J Optim 2011;21:1109-40.

[14] Silva ECN, Kikuchi N. Design of piezoelectric transducers using topology optimization. Smart Mater Struct 1999;8:350-64.

[15] Kögl M, Silva ECN. Topology optimization of smart structures: design of piezoelectric plates and Shell actuators. Smart Mater Struct 2005;14:387-99.

[16] Goldberg DE. Genetic algorithms in search, optimization and machine learning. Addison-Wesley; 1989.

[17] Yang G, Reinstein LE, Pai S, Xu Z, Carroll DL. A new genetic algorithm technique in optimization of permanent 125-1 prostate implants. Med Phys 1998;25:2308-15. 